\title{
The impact of the COVID-19 pandemic on oncological disease extent at FDG PET/CT staging: the ONCOVIPET study
}

\author{
Carmelo Caldarella ${ }^{1} \cdot$ Fabrizio Cocciolillo ${ }^{2}$ Silvia Taralli ${ }^{1} \cdot$ Margherita Lorusso $^{2} \cdot$ Valentina Scolozzi $^{1}$. \\ Daniele Antonio Pizzuto ${ }^{1}$ Maria Lucia Calcagni ${ }^{1,3} \cdot$ Vittoria Rufini $^{1,3} \cdot$ Davide Guido $^{4} \cdot$ Fernando Palluzzi $^{4}$. \\ Luciano Giacò $^{4} \cdot$ Alessandro Giordano $^{1,3} \cdot$ Lucia Leccisotti $^{1,3,4}(\mathbb{0}$
}

Received: 26 August 2021 / Accepted: 20 November 2021 / Published online: 8 December 2021

(c) The Author(s), under exclusive licence to Springer-Verlag GmbH Germany, part of Springer Nature 2021

\begin{abstract}
Purpose To investigate whether the COVID-19 pandemic and national lockdown had an impact on the extent of cancer disease at FDG PET/CT staging as surrogate marker.

Methods Retrospective observational study including cancer patients submitted to FDG PET/CT staging from June 1 to October 31, 2020, and June 1 to October 31, 2019, respectively. Data regarding primary tumour, nodal (N) status and number of involved nodal stations, and presence and number of distant metastases (M) were collected. Each scan was classified in limited vs advanced status. Data were aggregated across the study population and tumour type. Bi-weekly frequencies of the observed events were analysed.

Results Six hundred eleven patients were included (240 in 2019 vs 371 in 2020, respectively). A significant increase of advanced disease patients (rate 1.56, $P<0.001$ ), $\mathrm{N}+$ or $\mathrm{M}+$ patients (rate 1.84 and 2.09, respectively, $P<0.001$ ), and patients with a greater number of involved $\mathrm{N}$ stations or $\mathrm{M}$ (rate 2.01 and 2.06, respectively, $P<0.001$ ) were found in 2020 compared with data of 2019. Analysis by tumour type showed a significant increase of advanced disease in lymphoma and lung cancer in 2020 compared with $2019(P<0.001)$. In addition, a significant increase of nodal involvement was found in lung, gastrointestinal, and breast cancers, as well as in lymphoma patients $(P<0.02)$. A significant increase of distant metastases was found in lung cancers $(P=0.002)$.

Conclusion Cancer patients with advanced disease at FDG PET/CT staging increased in 2020 compared with 2019, following the national lockdown due to the COVID-19 pandemic, 1.5-fold with a significant increase of patients with $\mathrm{N}$ or $\mathrm{M}$ involvement. Targeted health interventions are needed to mitigate the effects of the pandemic on patient outcome.
\end{abstract}

Keywords COVID-19 $\cdot$ FDG $\cdot$ Oncology $\cdot$ Pandemic $\cdot$ PET $\cdot$ Staging

This article is part of the Topical Collection on Oncology General

Lucia Leccisotti

lucia.leccisotti@policlinicogemelli.it

1 Dipartimento di Diagnostica per Immagini, Radioterapia Oncologica ed Ematologia, UOC di Medicina Nucleare, Fondazione Policlinico Universitario Agostino Gemelli IRCCS, Rome, Italy

2 PET/CT Center, Fondazione Policlinico Universitario Agostino Gemelli IRCCS, Rome, Italy

3 Dipartimento Universitario di Scienze Radiologiche ed Ematologia, Sezione di Medicina Nucleare, Università Cattolica del Sacro Cuore, Rome, Italy

4 Fondazione Policlinico Universitario Agostino Gemelli IRCCS, Largo A. Gemelli, 8, 00168 Rome, Italy

\section{Introduction}

The global coronavirus disease 2019 (COVID-19) pandemic has severely affected healthcare systems, economy, work, education, and social relationships. On March 2020, National Health Services (NHSs) of many countries, starting with Italy, suddenly redesigned their services to increase the capacity for treating patients with COVID-19. Procedures included discharging thousands of inpatients to free up beds, postponing scheduled treatments, shifting appointments online whenever possible, and redeploying healthcare staff. In addition, on March 9, 2020, the Italian Government declared a national lockdown in response to the COVID-19 pandemic, which lasted until May 18, 2020. "Stay-at-home" orders were enacted to slow the spread of 
the severe acute respiratory syndrome coronavirus 2 (SARS$\mathrm{CoV}-2$ ) and reduce the burden on the NHS. Consequently, the COVID-19 emergency had direct and indirect effects on the healthcare delivery process. Direct effects included the radical reorganization of health systems to respond to the acute needs of people infected with the SARS-CoV-2 and to contain the infection protecting the most vulnerable categories and healthcare personnel. Indirect effects of the pandemic are represented by the impact on people with acute and non-acute conditions not related to COVID19, especially cardiovascular and oncological diseases [1-7]. In oncology, the best outcomes are obtained in those tumours diagnosed at an early stage and treatment is started straight away. Unfortunately, several diagnostic and treatment pathways, used for cancer patient management, have been severely affected, especially during the "first wave" of the COVID-19 pandemic [8-11]. In addition, the fear of COVID-19 transmission contributed to a lower propensity of patients with symptoms to refer directly to hospitals. Therefore, many patients likely experienced a delay in both diagnosis and treatment of cancer. To date, no direct population-based evidence of the impact of the COVID-19 pandemic on the extent of cancer disease at staging is available. Positron emission tomography/computed tomography with 2-deoxy-2-[18F] fluoro-D-glucose (FDG PET/CT) has become one of the cornerstones of patient management in oncology. The high diagnostic accuracy of FDG PET/CT at staging to detect lymph node and distant metastasis and the impact on patient management has been widely reported in several malignancies [12-24]. This study aims to evaluate whether the delay in cancer diagnosis and initial staging, due to the COVID-19 pandemic and its consequent national lockdown, had an impact on disease extent in cancer patients using FDG PET/CT staging as surrogate measure.

\section{Materials and methods}

\section{Population, selection criteria, and data extraction}

We conducted a single-centre retrospective observational study including all consecutive oncological patients submitted to whole-body FDG PET/CT staging at the PET-CT Centre of the Fondazione Policlinico Universitario Agostino Gemelli IRCCS in Rome, Italy, in two selected intervals: June 1 to October 31, 2019, and June 1 to October 31, 2020. These intervals were selected to evaluate effects of any diagnostic delays on disease extent at time of initial staging due to the first pandemic wave and the restrictions enforced during the national lockdown from March 9 to May 18, 2020. This study was approved by the local ethics committee (No. 4024), and informed consent was obtained from all patients included in the study. Patients' information was retrieved from internal medical records and PET/CT images. Only patients $\geq 18$ years of age with a cancer diagnosis and FDG-avid tumours, who performed a whole-body PET/CT for staging purposes, were included in the study. Patients performing PET/CT for restaging purposes (e.g., suspected recurrence, therapy response evaluation) or with radiopharmaceuticals other than FDG were excluded. FDG PET/CT images of all included patients were retrieved from the Institution's Picture Archive and Communication System and displayed on a dedicated workstation using the software Syngo.via (Siemens ${ }^{\circledR}$ ). All included patients were divided into sub-groups according to the primary tumour (i.e., lung, head and neck, gynaecologic, gastro-intestinal, breast, lymphoma, melanoma, and myeloma). PET/CT images were evaluated independently by 2 nuclear medicine physicians for each tumour sub-group referring to the report previously drawn up to assess the disease stage according to the International Classification of Diseases for Oncology (American Joint Committee on Cancer staging manual) [25]. Information regarding patients' gender, age, date of PET/CT scan, site, and histology of primary tumour (T), presence/absence of lymph node $(\mathrm{N}+/ \mathrm{N} 0)$ involvement and number of involved nodal stations, presence/absence of distant metastases $(\mathrm{M}+/ \mathrm{M} 0)$, and number of metastatic lesions was collected. Myeloma patients were excluded from nodal assessment because of the very rare nodal spreading of the disease. In lymphoma patients, all extranodal disease sites were considered metastases for statistical analysis. Based on the number of distant metastases, including extranodal disease sites in lymphomas, a 4-point scale -0 (no distant metastases), 1 (1 to 3 lesions), 2 (4 to 7 lesions), and 3 (>7 lesions)—was used. Based on this evaluation, all PET/ CT scans were categorized in advanced or limited disease stages: advanced stage (III and IV) or limited stage (I and II) for breast, gynaecologic, lung, and head and neck cancers, lymphoma, and melanoma; advanced stage (any T, any N, M1) or limited stage (M0) for gastro-intestinal cancers; and advanced stage (extra-skeletal disease and/or presence of lytic lesions on CT) or limited stage (no extra-skeletal disease, no lytic lesions on CT) for myeloma. Additionally, to confirm PET tumour staging, information on the subsequent patient management was reviewed (e.g., medical records referred to tumour multidisciplinary boards, subsequent examinations performed, type of treatment).

\section{Statistical analysis}

Data were gathered in the study population and tumour type generating bi-weekly frequencies for the observed $\mathrm{T}$, $\mathrm{N}$, and $\mathrm{M}$ events, the number of $\mathrm{N}$ stations and $\mathrm{M}$ sites, and tumours in advanced status. This was done because the updates of the national health surveillance were provided bi-weekly and the standard quarantine period covered a 
15-day period. Poisson models were fitted to investigate how the years 2019 vs 2020 affected the observed frequencies. For each model, the year was used as a binary predictor, and the exponential transformation of the coefficient was interpreted as rate. Of note, this study has not analysed the incidence of tumour or advance disease. In fact, the Poisson models have been fitted without the offset term and returned coefficients (in exponential terms) interpretable as rate. We assumed that hospital user base across the year is very similar. Finally, myeloma and head and neck tumours were analysed by Firth's logistic regression modelling [26] or small samples because of very low or null counts in response variables. In these cases, binary outcomes were considered $(0=$ counts null, $1=$ counts not null), and the exponential transformation of the coefficient was interpreted as odds ratio. $P$ values less than 0.05 indicated significance. All the analyses were performed using $\mathrm{R}$ statistical environment (version 4.0.3).

\section{Results}

Three thousand five hundred fifty-one and 3443 FDG PET/ CT scans were performed in our centre from June 1 to October 31, 2019, and from June 1 to October 31, 2020, respectively (Fig. 1). According to the inclusion and exclusion criteria, 240 patients in 2019 and 371 patients in 2020 were finally included in the study. The main characteristics of study population are described in Table 1 . Table 2 shows the bi-weekly frequencies for the observed $\mathrm{T}, \mathrm{N}+$, and $\mathrm{M}+$ events, the number of $\mathrm{N}$ stations and $\mathrm{M}$ sites, and tumours in advanced status over the two reference periods.
Table 1 Study population characteristics $(n=611)$

\begin{tabular}{lll}
\hline & $\begin{array}{l}\text { June 1-October 31, } \\
2019\end{array}$ & $\begin{array}{l}\text { June 1- } \\
\text { October 31, } \\
2020\end{array}$ \\
\hline$n$ & 240 & 371 \\
Age (yrs), mean \pm SD & $63 \pm 14$ & $63 \pm 15$ \\
Male, $n(\%)$ & $106(44)$ & $157(42)$ \\
Tumour type, $n(\%)$ & & \\
Lung & $75(31.3)$ & $112(30.2)$ \\
Gynaecologic & $53(22.1)$ & $76(20.5)$ \\
Gastro-intestinal & $36(15)$ & $47(12.7)$ \\
Lymphoma & $32(13.3)$ & $67(18.1)$ \\
Breast & $16(6.7)$ & $26(7)$ \\
Head \& neck & $15(6.2)$ & $20(5.4)$ \\
Myeloma & $10(4.2)$ & $12(3.1)$ \\
Melanoma & $3(1.2)$ & $11(3)$ \\
\hline
\end{tabular}

$n$ frequency, $S D$ standard deviation

\section{Advanced vs limited disease at FDG PET/CT staging}

Overall, the bi-weekly analysis of ranges showed a significant increase in the rate of advanced tumours in 2020 compared with 2019 (rate 1.56; 95\% confidence interval [CI] $1.23-1.97 ; P<0.001)$.

\section{Nodal and metastatic disease at FDG PET/CT staging}

Overall, the bi-weekly analysis demonstrated a significant increase in the rate of $\mathrm{N}+$ or $\mathrm{M}+$ patients in 2020 compared with $2019(\mathrm{~N}+$ : rate $1.85,95 \% \mathrm{CI} 1.50-2.27, P<0.001 ; \mathrm{M}+$ : rate $2.09,95 \% \mathrm{CI} 1.55-2.82, P<0.001)$. The rate of patients
Fig. 1 Flowchart of FDG PET/ CT scans from June 1 to October 31, 2019, and from June 1 to October 31, 2020

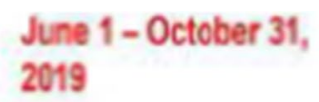

June 1-October 31, 2020

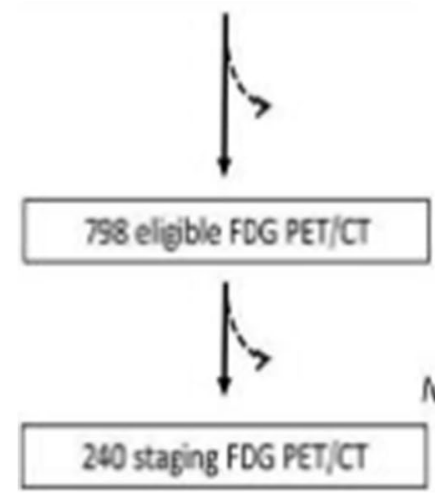

\section{FOG PET/CT}

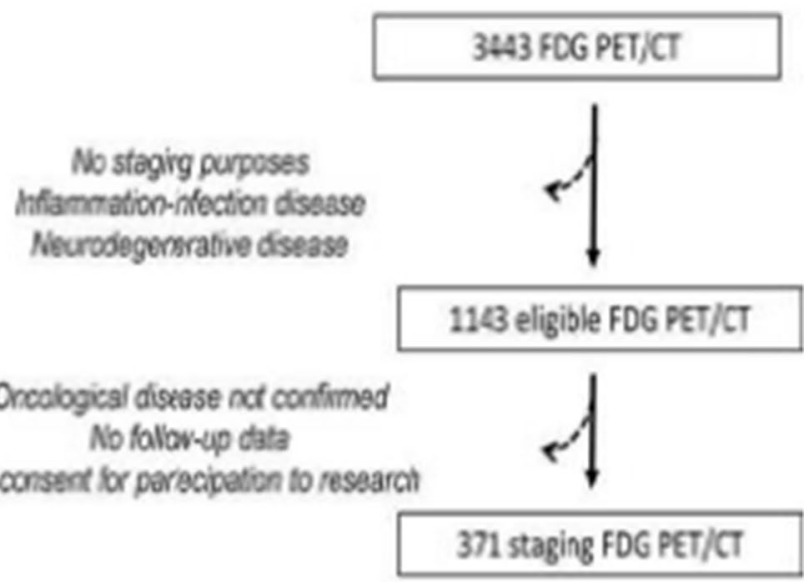


Table 2 Bi-weekly frequencies (11 intervals) for the observed $\mathrm{T}, \mathrm{N}+$, and $\mathrm{M}+$ events, the number of $\mathrm{N}$ stations and $\mathrm{M}$ lesions, and tumours in advanced status in 2019 and 2020

\begin{tabular}{|c|c|c|c|c|c|c|}
\hline 2019 intervals & $\mathrm{T}^{\#}$ & $\mathrm{~N}+$ & $\mathrm{M}+$ & $\mathrm{N}$ stations & M sites* & Advanced disease \\
\hline 1 & 34 & 25 & 10 & 111 & 16 & 21 \\
\hline 2 & 24 & 12 & 8 & 51 & 20 & 15 \\
\hline 3 & 11 & 11 & 5 & 42 & 10 & 8 \\
\hline 4 & 17 & 13 & 7 & 38 & 10 & 10 \\
\hline 5 & 16 & 10 & 5 & 23 & 9 & 8 \\
\hline 6 & 18 & 12 & 4 & 29 & 6 & 12 \\
\hline 7 & 19 & 13 & 10 & 75 & 13 & 13 \\
\hline 8 & 10 & 10 & 5 & 43 & 8 & 7 \\
\hline 9 & 20 & 13 & 4 & 21 & 5 & 7 \\
\hline 10 & 10 & 12 & 4 & 67 & 4 & 7 \\
\hline 11 & 8 & 7 & 2 & 17 & 2 & 6 \\
\hline 2020 intervals & $\mathrm{T}^{\#}$ & $\mathrm{~N}+$ & $\mathrm{M}+$ & $\mathrm{N}$ stations & M sites* & Advanced disease \\
\hline 1 & 14 & 19 & 8 & 67 & 14 & 11 \\
\hline 2 & 27 & 21 & 12 & 93 & 23 & 16 \\
\hline 3 & 28 & 25 & 11 & 92 & 21 & 18 \\
\hline 4 & 26 & 23 & 9 & 81 & 10 & 17 \\
\hline 5 & 26 & 25 & 14 & 101 & 24 & 20 \\
\hline 6 & 25 & 20 & 10 & 92 & 17 & 14 \\
\hline 7 & 21 & 25 & 17 & 151 & 23 & 19 \\
\hline 8 & 25 & 20 & 10 & 74 & 13 & 15 \\
\hline 9 & 28 & 25 & 15 & 103 & 27 & 15 \\
\hline 10 & 30 & 25 & 14 & 76 & 21 & 14 \\
\hline 11 & 25 & 27 & 14 & 107 & 20 & 19 \\
\hline
\end{tabular}

${ }^{\#} \mathrm{~T}$ cases represent solid tumours only; *extranodal sites of lymphoma patients were included in the M analysis as reported in the "Materials and methods" section with a higher number of involved $\mathrm{N}$ or $\mathrm{M}$ stations resulted significantly increased in 2020 compared with 2019 (rate of N stations: $2.01,95 \%$ CI 1.80-2.23, $P<0.001$; rate of $\mathrm{M}$ sites: 2.06, 95\% CI 1.63-2.61, $P<0.001)$.

\section{Analysis by tumour type}

The bi-weekly frequency analysis demonstrated a significant increase in advanced disease, $\mathrm{N}+$ and $\mathrm{M}+$ /extranodal rate, and number of involved $\mathrm{N}$ stations and $\mathrm{M}$ sites for both lung cancer and lymphoma in 2020 compared with 2019 (Table 3). Compared with 2019, advanced disease rate significantly decreased in 2020 in gynaecologic cancer. Nodal involvement resulted increased in gastro-intestinal and breast cancers. Figures showing bi-weekly frequency distribution of advanced disease status, $\mathrm{N}+, \mathrm{M}+$, and number of nodal stations involved and distant metastases at FDG PET/CT staging are provided in the Supplementary Material (Fig. 2-6).

\section{Discussion}

The results of our study show that the rate of cancer patients with advanced disease at time of FDG PET/CT staging was 1.5-fold higher in 2020 compared with 2019, following the national lockdown due to the COVID-19 pandemic. In addition, we found approximately a twofold increase at staging in the rate of cancer patients with nodal involvement or metastatic disease and with a greater number of involved nodes or distant metastases. In 2020 compared with 2019, patients with lung cancer and lymphoma showed a significant (1.9 to 2.6-fold) increase at staging in advanced disease rate, nodal/extranodal involvement, metastatic status, and number of nodal stations and metastatic/extranodal sites. In addition, gastro-intestinal and breast cancers showed a significant increase of nodal involvement in 2020 compared with 2019. However, gynaecological cancers showed a significant decrease in advanced disease rate at staging in 2020 vs 2019. This retrospective observational study indirectly analysed the impact of the first COVID-19 pandemic wave and its national lockdown on the extent of cancer disease at staging using whole-body FDG PET/CT as a surrogate marker. Oncological FDG PET/CT is known to be the most accurate non-invasive technique for cancer staging in most histotypes because of its superiority over radiological imaging, mainly in assessing lymph node involvement and distant metastases [12]. From our data, more cancer patients showed an advanced disease stage at time of FDG PET/CT staging in 2020, after the national lockdown, compared with the same reference period in 2019. An increased rate of patients with 
Table 3 Bi-weekly analysis results for tumour type (2020 vs 2019)

\begin{tabular}{|c|c|c|c|}
\hline & Rate & $95 \% \mathrm{CI}$ & $P$ value \\
\hline \multicolumn{4}{|l|}{ Lung cancer } \\
\hline Advanced stage & 1.92 & $1.3-2.8$ & $<0.001$ \\
\hline $\mathrm{N}+$ & 1.97 & $1.3-2.9$ & $<0.001$ \\
\hline$M+$ & 2.25 & $1.3-3.8$ & 0.0025 \\
\hline $\mathrm{N}(\mathrm{n})$. & 2.30 & $1.8-2.8$ & $<0.001$ \\
\hline $\mathrm{vM}(\mathrm{n})$. & 2.55 & $1.7-3.8$ & $<0.001$ \\
\hline \multicolumn{4}{|l|}{ Lymphoma } \\
\hline Advanced stage & 2.68 & $1.5-4.8$ & $<0.001$ \\
\hline $\mathrm{N}+$ & 2.42 & $1.5-3.8$ & $<0.001$ \\
\hline Extra nodal+ & 2.31 & $1.3-3.9$ & 0.0022 \\
\hline $\mathrm{N}(\mathrm{n})$. & 2.15 & $1.8-2.5$ & $<0.001$ \\
\hline Extra nodal (n.) & 2.59 & $1.5-4.3$ & $<0.001$ \\
\hline \multicolumn{4}{|c|}{ Gastro-intestinal cancer } \\
\hline Advanced stage & 1.63 & $0.7-3.4$ & 0.1981 \\
\hline $\mathrm{N}+$ & 1.99 & $1.1-3.5$ & 0.0163 \\
\hline $\mathrm{M}+$ & 1.62 & $0.6-3.9$ & 0.2799 \\
\hline $\mathrm{N}(\mathrm{n})$. & 1.68 & $1.1-2.4$ & 0.0053 \\
\hline$M(n)$. & 1.49 & $0.7-2.8$ & 0.2090 \\
\hline \multicolumn{4}{|l|}{ Breast cancer } \\
\hline Advanced stage & 1.24 & $0.4-3.1$ & 0.6380 \\
\hline $\mathrm{N}+$ & 1.90 & $0.9-3.9$ & 0.0823 \\
\hline $\mathrm{M}+$ & 3.49 & $0.7-16.8$ & 0.1181 \\
\hline $\mathrm{N}(\mathrm{n})$. & 2.66 & $1.6-4.4$ & 0.0001 \\
\hline M (n.) & 2.99 & $0.9-9.3$ & 0.0570 \\
\hline \multicolumn{4}{|l|}{ Gynaecologic cancer } \\
\hline Advanced stage & 0.48 & $0.2-0.8$ & 0.0209 \\
\hline $\mathrm{N}+$ & 1.45 & $0.9-2.2$ & 0.1103 \\
\hline$M+$ & 2.19 & $0.7-6.3$ & 0.1437 \\
\hline $\mathrm{N}(\mathrm{n})$. & 1.31 & $0.9-1.7$ & 0.0576 \\
\hline M (n.) & 0.99 & $0.4-2.3$ & 0.9999 \\
\hline \multicolumn{4}{|l|}{ Head \& neck tumour } \\
\hline Advanced stage & $0.68 *$ & $0.1-4.1$ & 0.6790 \\
\hline $\mathrm{N}+$ & $0.63 *$ & $0.1-4.5$ & 0.1103 \\
\hline $\mathrm{M}+$ & $0.50 *$ & $0.1-2.8$ & 0.4350 \\
\hline $\mathrm{N}(\mathrm{n})$. & $0.63 *$ & $0.1-4.5$ & 0.6529 \\
\hline$M(n)$. & $0.50 *$ & $0.1-2.8$ & 0.4350 \\
\hline \multicolumn{4}{|l|}{ Myeloma } \\
\hline Advanced stage & $2.28 *$ & $0.3-15$ & 0.3950 \\
\hline $\mathrm{M}+$ & $2.87 *$ & $0.5-16.7$ & 0.2402 \\
\hline M (n.) & $2.87 *$ & $0.5-16.7$ & 0.2402 \\
\hline
\end{tabular}

$N+$ presence of nodal involvement, $M+$ presence of metastatic disease; $N(n$.) number of nodal stations, $M(n$.) number of metastatic sites grouped using a 4-point scale ( 0 [no distant metastases], 1 [1 to 3 lesions], 2 [4 to 7 lesions] and 3 [> 7 lesions]; *Odds ratio obtained by Firth's logistic regression models. In bold are the significant results $(P<0.05)$

The rates (or odds ratio), 95\% CIs, and $P$-values are returned by Poisson or Firth's logistic regression modelling, applied to the bi-weekly data (i.e., statistical units). The predictor of the statistical models is the binary variable regarding the year (2019 vs 2020) nodal involvement and metastatic disease was also demonstrated. This increase might be explained with delays in accessing cancer diagnosis for many patients correlated to the COVID-19 pandemic. The first phase of the emergency determined a reduction in inpatient and outpatient services provided to non-COVID patients, i.e. delays or suspensions of important screening programmes, limitations of some diagnostic tests such as CTs in the emergency context and cancellations or delays of specialist visits, and some elective procedures such as biopsies or surgeries [27-31]. Several European countries reported a drop of 15 to $40 \%$ of diagnostic cancer rates at the beginning of the pandemic. In Italy, approximately 1.4 million fewer screening exams were performed in the first 5 months of 2020 compared with the same period in 2019 [32]. Surgery was the most affected modality having been delayed or cancelled in over $10 \%$ of patients in $34 \%$ of European Centres [11]. In Italy, a significant decrease (20-50\%) in the volumes of screening tests, outpatient specialist visits, and oncological interventions was reported in 2020 compared with 2019, even in geographical areas where a lower incidence of the SARS-CoV-2 was recorded during the first wave of the pandemic [32]. In addition to limited diagnostic capacity and patient admissions, many patients were reluctant to visit hospitals out of fear being infected or they preferred to postpone elective care according to public health measures encouraging people to stay at home. The quarantine and financial difficulties due to the lockdown had also an impact on travel and care-seeking behaviours. In fact, the incidence of cancer has not changed in recent years, and, in our context, the number of PET exams performed at our centre has not significantly changed in 2020 compared with 2019 as well as the planning of staging FDG PET/CT exams, which are generally performed within 7 days of receiving the request form. We have only recorded a slight reduction in PET/CT requests for patients on treatment due to cancellation or postponement of the examination with a consequent greater availability to perform examinations for staging or restaging purposes. Given that the incidence of cancer has not significantly changed in recent years, the increase in the number of FDG PET/CT requests for staging purposes immediately after the national lockdown is most likely due to the delay of cancer diagnosis in many patients due to the restrictions imposed by the government and hospital saturation due to COVID patients. Delays in cancer diagnosis led to more patients being diagnosed at a more advanced stage of their disease, as demonstrated by our study. This means that these patients might require more complex treatments than otherwise needed and that more deaths result from cancer. The exact extent of the effects on survival and healthcare costs will only become evident in the next few years. After the first peak of the pandemic, forecasts on the impact of these delays on cancer diagnosis and patient survival were provided. The severity of the indirect effects of 
the COVID-19 pandemic was clear in November 2020 when a 4-week treatment delay was reported to be associated with a 6 to $17 \%$ increased risk of death depending on cancer type. Delays of up to 12 weeks increase this risk further [33-36]. However, the challenge is to prevent that new waves of the pandemic limit access to cancer diagnosis and treatment, by constructing a health system which adequately responds to the needs of these advanced stage patients both during and shortly after the pandemic. We hope that the detrimental impact of the COVID-19 pandemic on cancer patients could be mitigated with the support of all stakeholders in cancer diagnosis, treatment, and health planning processes. This study has some limitations. First, this is a retrospective study with its intrinsic limitations, which, however, indirectly allowed us to take a snapshot of real events related to this emergency. Secondly, we focused on patients with FDGavid tumours excluding other malignancies such as prostate cancer or neuroendocrine tumours that are usually studied with radiopharmaceuticals other than FDG. Nevertheless, FDG is the radiopharmaceutical most frequently used for PET, and FDG-avid tumours are the most aggressive cancer types for which a delay in diagnosis and treatment initiation could have the worst consequences on patients' outcome [37]. In addition, considering the subset of patients studied (with cancers at staging), it is unlikely that the results are biased by the inclusion of data from the summer months of 2019, which were vacation months. Conversely, the obtained results (rates in particular) are likely due to the saturated hospitality capacity during the COVID-19 outbreak. Finally, we did not find any statistically significant difference in myeloma and head and neck tumours most likely due to the low sample size. Further, more complex, studies addressed to evaluate patient outcome, survival, and healthcare costs are desirable to confirm our results.

\section{Conclusion}

The COVID-19 pandemic has had a huge and negative impact on the diagnosis and initiation of cancer treatment. Overall, the rate of oncological patients with advanced disease stage at time of FDG PET/CT staging increased 1.5fold in 2020 compared with 2019 with a twofold increase of patients with nodal involvement and distant metastases. Targeted health interventions are needed to mitigate the expected impact of the COVID-19 pandemic on the outcome of cancer patients.

Supplementary Information The online version contains supplementary material available at https://doi.org/10.1007/s00259-021-05629-0.

Acknowledgements We thank the medical technician Marco De Summa (Medipass Advanced Medical Services spa) for his valuable collaboration in material preparation.
The Bioinformatics Core Facility Research performed data analysis at the Gemelli Science and Technology Park (GSTeP).

We would like to thank Franziska M. Lohmeyer, PhD, Fondazione Policlinico Universitario A. Gemelli IRCCS, for her support revising our manuscript.

Author contribution All authors contributed to the study conception and design. Material preparation and data collection were performed by Carmelo Caldarella, Fabrizio Cocciolillo, Silvia Taralli, Margherita Lorusso, Valentina Scolozzi, Daniele Antonio Pizzuto, and Lucia Leccisotti. Data analysis was performed in the Gemelli Science and Technology Park (GSTeP), the Bioinformatics Core Facility Research, by Davide Guido, Fernando Palluzzi, and Luciano Giacò. The first draft of the manuscript was written by Carmelo Caldarella and Lucia Leccisotti, and all authors commented on previous versions of the manuscript. All authors read and approved the final manuscript.

Data availability The datasets generated during and/or analysed during the current study are available from the corresponding author on reasonable request.

\section{Declarations}

Ethics approval This retrospective observational study involving human participants was in accordance with the ethical standards of the institutional research committee and with the 1964 Helsinki Declaration and its later amendments or comparable ethical standards. The ethics committee of the Fondazione Policlinico Universitario Agostino Gemelli IRCCS-Università Cattolica del Sacro Cuore approved this study (Date 15.4.2021/No. 4024).

Conflict of interest The authors declare no competing interests.

\section{References}

1. Patt D, Gordan L, Diaz M, Okon T, Grady L, Harmison M, et al. Impact of COVID-19 on cancer care: how the pandemic is delaying cancer diagnosis and treatment for American seniors. JCO Clin Cancer Inform. 2020;4:1059-71. https://doi.org/10.1200/ CCI.20.00134.

2. de Jonge L, Worthington J, van Wifferen F, Iragorri N, Peterse EFP, Lew JB, et al. Impact of the COVID-19 pandemic on faecal immunochemical test-based colorectal cancer screening programmes in Australia, Canada, and the Netherlands: a comparative modelling study. Lancet Gastroenterol Hepatol. 2021. https:// doi.org/10.1016/S2468-1253(21)00003-0.

3. Oderda M, Calleris G, Falcone M, Fasolis G, Muto G, Oderda G, et al. How uro-oncology has been affected by COVID-19 emergency? Data from Piedmont/Valle d'Aosta Oncological Network. Ital Urol. 2021;88:3-8. https://doi.org/10.1177/0391560320 946186.

4. Fabi A, Pugliese P, Falbo PT, Corsi D, Fabbri MA, Vincenzi B, et al. The experience of oncology healthcare providers in the Central Italy during the COVID-19 lockdown. Cancers (Basel). 2020;12:3031. https://doi.org/10.3390/cancers12103031.

5. Quaquarini E, Saltalamacchia G, Presti D, Caldana G, Tibollo V, Malovini A, et al. Impact of COVID-19 outbreak on cancer patient care and treatment: data from an outpatient oncology clinic in Lombardy (Italy). Cancers (Basel). 2020;12:2941. https://doi. org/10.3390/cancers 12102941.

6. Hirschfeld CB, Shaw LJ, Williams MC, Lahey R, Villines TC, Dorbala $\mathrm{S}$, et al. Impact of COVID-19 on cardiovascular testing in the 
United States versus the rest of the world: The INCAPS-COVID Study. JACC Cardiovasc Imaging. 2021. https://doi.org/10.1016/j. jcmg.2021.03.007.

7. Saini KS, de Las HB, de Castro J, Venkitaraman R, Poelman M, Srinivasan G, et al. Effect of the COVID-19 pandemic on cancer treatment and research. Lancet Haematol. 2020;7:e432-5. https:// doi.org/10.1016/S2352-3026(20)30123-X.

8. Di Bidino R, Cicchetti A. Impact of SARS-CoV-2 on provided healthcare. Evidence From the Emergency Phase in Italy. Front Public Health. 2020;8:583583. https://doi.org/10.3389/fpubh.2020.583583.

9. Torzilli G, Viganò L, Galvanin J, Castoro C, Quagliuolo V, Spinelli A, et al. A snapshot of elective oncological surgery in Italy during COVID-19 emergency: pearls, pitfalls, and perspectives. Ann Surg. 2020;272:e112-7. https://doi.org/10.1097/SLA.0000000000004081.

10. Albano D, Bruno A, Bruno F, Calandri M, Caruso D, Clemente A, et al. Impact of coronavirus disease 2019 (COVID-19) emergency on Italian radiologists: a national survey. Eur Radiol. 2020;30:663544. https://doi.org/10.1007/s00330-020-07046-7.

11. Onesti CE, Tagliamento M, Curigliano G, Harbeck N, Bartsch R, Wildiers $\mathrm{H}$, et al. Expected medium- and long-term impact of the COVID-19 outbreak in oncology. JCO Glob Oncol. 2021;7:162-72. https://doi.org/10.1200/GO.20.00589.

12 Boellaard R, Delgado-Bolton R, Oyen WJ, Giammarile F, Tatsch $\mathrm{K}$, Eschner W, et al. FDG PET/CT: EANM procedure guidelines for tumour imaging: version 2.0. Eur J Nucl Med Mol Imaging. 2015;42(328):54. https://doi.org/10.1007/s00259-014-2961-x.

13 Kostakoglu L, Agress H, Goldsmith SJ. Clinical role of FDG PET in evaluation of cancer patients. Radiographics. 2003;23:315-40. https://doi.org/10.1148/rg.232025705 (quiz 533).

14. Kandathil A, Kay FU, Butt YM, Wachsmann JW, Subramaniam RM. Role of FDG PET/CT in the Eighth Edition of TNM Staging of Non small Cell Lung Cancer. Radiographics. 2018;38:2134-49. https://doi.org/10.1148/rg.2018180060.

15. Wartski M, Sauvanet A. 18F-FDG PET/CT in pancreatic adenocarcinoma: a role at initial imaging staging? Diagn Interv Imaging. 2019;100:735-41. https://doi.org/10.1016/j.diii.2019.07.006.

16. Paydary K, Seraj SM, Zadeh MZ, Emamzadehfard S, Shamchi SP, Gholami S, et al. The evolving role of FDG-PET/CT in the diagnosis, staging, and treatment of breast cancer. Mol Imaging Biol. 2019;21:1-10. https://doi.org/10.1007/s11307-018-1181-3.

17. Ha HK, Koo PJ, Kim SJ. Diagnostic accuracy of F-18 FDG PET/ CT for preoperative lymph node staging in newly diagnosed bladder cancer patients: a systematic review and meta-analysis. Oncology. 2018;95:31-8. https://doi.org/10.1159/000488200.

18. CaresiaAroztegui AP, García Vicente AM, Alvarez Ruiz S, Delgado Bolton RC, Orcajo Rincon J, Garcia Garzon JR, et al. 18F-FDG $\mathrm{PET} / \mathrm{CT}$ in breast cancer: evidence-based recommendations in initial staging. Tumour Biol. 2017;39:1010428317728285. https://doi.org/ $10.1177 / 1010428317728285$.

19. Thanarajasingam G, Bennani-Baiti N, Thompson CA. PET-CT in staging, response evaluation, and surveillance of lymphoma. Curr Treat Options Oncol. 2016;17:24. https://doi.org/10.1007/ s11864-016-0399-z.

20. Perng P, Marcus C, Subramaniam RM. (18)F-FDG PET/CT and melanoma: staging, immune modulation and mutation-targeted therapy assessment, and prognosis. AJR Am J Roentgenol. 2015;205:259-70. https://doi.org/10.2214/AJR.14.13575.

21. Jones M, Hruby G, Solomon M, Rutherford N, Martin J. The role of FDG-PET in the initial staging and response assessment of anal cancer: a systematic review and meta-analysis. Ann Surg Oncol. 2015;22:3574-81. https://doi.org/10.1245/s10434-015-4391-9.

22. Kaneko Y, Murray WK, Link E, Hicks RJ, Duong C. Improving patient selection for 18F-FDG PET scanning in the staging of gastric cancer. J Nucl Med. 2015;56:523-9. https://doi.org/10.2967/jnumed. 114.150946.
23. Mak D, Corry J, Lau E, Rischin D, Hicks RJ. Role of FDG-PET/CT in staging and follow-up of head and neck squamous cell carcinoma. Q J Nucl Med Mol Imaging. 2011;55:487-99.

24. Yousefi-Koma A, Panah-Moghaddam M, Kalff V. The utility of metabolic imaging by $18 \mathrm{~F}-\mathrm{FDG}$ PET/CT in lung cancer: impact on diagnosis and staging. Tanaffos. 2013;12:16-25.

25. Amin MB, Edge SB, Greene FL, Byrd DR, Brookland RK, Washington MK, Gershenwald JE, Compton CC, Hess KR, Sullivan DC, Jessup JM, Brierley JD, Gaspar LE, Schilsky RL, Balch CM, Winchester DP, Asare EA, Madera M, Gress DM, Meyer LR (editors). AJCC cancer staging manual. 8th ed. Springer International Publishing; 2017.

26. Wang X. Firth logistic regression for rare variant association tests. Front Genet. 2014;5:187. https://doi.org/10.3389/fgene.2014.00187.

27. Jereczek-Fossa BA, Pepa M, Marvaso G, Bruni A, Buglione di Monale E, Bastia M, Catalano G, et al. COVID-19 outbreak and cancer radiotherapy disruption in Italy: survey endorsed by the Italian Association of Radiotherapy and Clinical Oncology (AIRO). Radiother Oncol. 2020;149:89-93. https://doi.org/10.1016/j.radonc. 2020.04.061.

28. Oldani C, Vanni G, Buonomo OC. COVID-19 unintended effects on breast cancer in Italy after the great lockdown. Front Public Health. 2020;8: 601748. https://doi.org/10.3389/fpubh.2020.601748.

29. Fortunato L, d'Amati G, Taffurelli M, Tinterri C, Marotti L, Cataliotti L. Severe impact of Covid-19 pandemic on breast cancer care in Italy: a Senonetwork national survey. Clin Breast Cancer. 2020. https://doi.org/10.1016/j.clbc.2020.10.012.

30. Rebecchi F, Arolfo S, Ugliono E, Morino M, Asti E, Bonavina L, et al. Impact of COVID-19 outbreak on esophageal cancer surgery in Northern Italy: lessons learned from a multicentric snapshot. Dis Esophagus. 2020. https://doi.org/10.1093/dote/doaa124.

31. Lambertini M, Toss A, Passaro A, Criscitiello C, Cremolini C, Cardone $\mathrm{C}$, Cancer care during the spread of coronavirus disease, et al. (COVID-19) in Italy: young oncologists' perspective. ESMO Open. 2019;2020:5. https://doi.org/10.1136/esmoopen-2020-000759.

32. https://www.agenas.gov.it/covid19/web/index.php?r=site\%2Fres ilienza. 2021.

33. Hanna TP, King WD, Thibodeau S, Jalink M, Paulin GA, HarveyJones E, et al. Mortality due to cancer treatment delay: systematic review and meta-analysis. BMJ. 2020;371: m4087. https://doi.org/ 10.1136/bmj.m4087.

34. Gheorghe A, Maringe C, Spice J, Purushotham A, Chalkidou K, Rachet B, et al. Economic impact of avoidable cancer deaths caused by diagnostic delay during the COVID-19 pandemic: a national population-based modelling study in England. UK Eur J Cancer. 2021. https://doi.org/10.1016/j.ejca.2021.04.019.

35. Maringe C, Spicer J, Morris M, Purushotham A, Nolte E, Sullivan R, et al. The impact of the COVID-19 pandemic on cancer deaths due to delays in diagnosis in England, UK: a national, population-based, modelling study. Lancet Oncol. 2020;21:1023-34. https://doi.org/ 10.1016/S1470-2045(20)30388-0.

36. Sud A, Torr B, Jones ME, Broggio J, Scott S, Loveday C, et al. Effect of delays in the 2-week-wait cancer referral pathway during the COVID-19 pandemic on cancer survival in the UK: a modelling study. Lancet Oncol. 2020;21:1035-44. https://doi.org/10.1016/ S1470-2045(20)30392-2.

37. Higashi K, Ueda Y, Ayabe K, Sakurai A, Seki H, Nambu Y, et al. FDG PET in the evaluation of the aggressiveness of pulmonary adenocarcinoma: correlation with histopathological features. Nucl Med Commun. 2000;21:707-14. https://doi.org/10.1097/00006231200008000-00002.

Publisher's note Springer Nature remains neutral with regard to jurisdictional claims in published maps and institutional affiliations. 